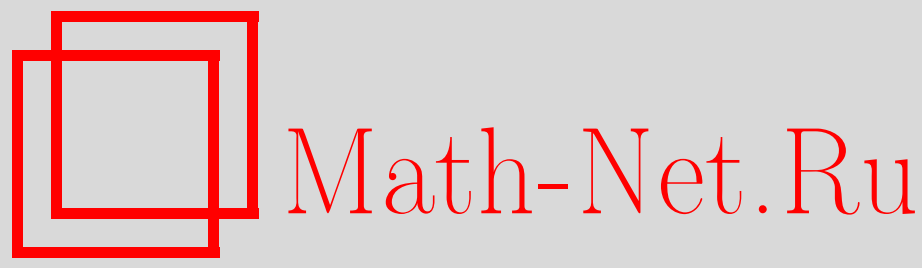

В. Ф. Лось, Н. В. Лось, Точное решение одномерного, зависящего от времени уравнения Шредингера с прямоугольной потенциальной ямой (барьером) и его применения, ТМФ, 2013, том 177, номер 3, 497-517

DOI: https://doi.org/10.4213/tmf8528

Использование Общероссийского математического портала Math-Net.Ru подразумевает, что вы прочитали и согласны с пользовательским соглашением http://www . mathnet.ru/rus/agreement

Параметры загрузки:

IP: 52.87 .193 .239

26 апреля 2023 г., $12: 43: 16$

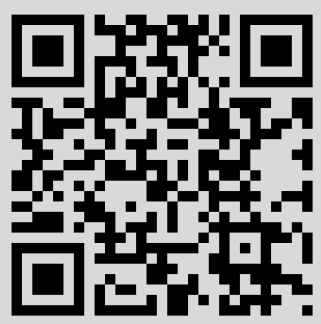




\title{
ТОЧНОЕ РЕШЕНИЕ ОДНОМЕРНОГО, ЗАВИСЯЩЕГО ОТ ВРЕМЕНИ УРАВНЕНИЯ ШРЕДИНГЕРА С ПРЯМОУГОЛЬНОЙ ПОТЕНЦИАЛЬНОЙ ЯМОЙ (БАРЬЕРОМ) И ЕГО ПРИМЕНЕНИЯ
}

\begin{abstract}
Получено точное одномерное, зависящее от времени решение для волновой функции $\psi(x, t)$ движущейся частицы в присутствии прямоугольной ямы (барьера). Решение, справедливое как для ямы, так и для барьера, определяется в терминах интегралов от элементарных функций и представляет собой сумму компонент волнового пакета, движущихся в прямом и обратном направлениях. Рассмотрены и численно визуализированы относительный вклад этих компонент и их интерференции в плотность вероятности $|\psi(x, t)|^{2}$, время прибытия и время пребывания частиц для узкого и широкого распределений по энергии (импульсу) в исходном гауссовом волновом пакете. Показано, что в случае широкого исходного волнового пакета существенным становится интуитивно неочевидный квантово-механический эффект влияния компонент, движущихся в обратном направлении, на рассматриваемые величины.
\end{abstract}

Ключевые слова: зависящее от времени уравнение Шредингера, прямоугольная потенциальная яма/барьер, движущиеся в обратном направлении компоненты волнового пакета, время пребывания, время прибытия.

DOI: $10.4213 / \operatorname{tmf} 8528$

\section{1. ВВЕДЕНИЕ}

Прямоугольные одномерные потенциалы представляют собой простейшие потенциалы, для которых можно исследовать некоторые необычные квантово-механические эффекты, такие как туннелирование частицы через потенциальный барьер или ее отражение от потенциальной ямы. Для стационарных состояний эти эффекты просто следуют из сшивки плоских волн и их производных на потенциальных ступеньках. Однако гораздо чаще имеет место более реалистичный случай, когда данные процессы зависят от времени, при этом частица, исходно локализованная вне потенциальной ямы (барьера), движется по направлению к потенциальной ступеньке и претерпевает рассеяние на потенциальных ступеньках. Для описания этих

*Институт магнетизма НАН Украины, Киев, Украина. E-mail: victorlos@mail.ru 
зависящих от времени процессов нужно решить соответствующее зависящее от времени уравнение Шредингера с прямоугольным потенциалом, что представляет собой достаточно непростую задачу.

Имеется еще один противоречащий интуиции (и часто упускаемый из виду) эффект даже в случае простейших одномерных, зависящих от времени задач. Волновой пакет, представляющий собой ансамбль частиц, исходно (при $t=t_{0}$ ) находящихся, скажем, где-то в области $x<0$, состоит как из положительных, так и из отрицательных компонент импульса, поскольку частица не может быть полностью локализована в области $x<0$, если волновой пакет содержит только компоненты с $p>0$. Можно было бы ожидать, что лишь частицы с положительными импульсами $p$ смогут достичь области с положительными координатами $x>0$ при $t>t_{0}$. Однако компоненты волнового пакета с отрицательными импульсами (ограниченные на полупрямую в импульсном пространстве) обязательно должны быть отличны от нуля во всем $x$-пространстве $(-\infty \div \infty)$. Они выражают присутствие частиц в области $x>0$ в начальный момент времени $t_{0}$ и поэтому могут давать вклад, например, в распределение времени прибытия частицы в область $x>0$ [1], [2]. Нелишне отметить, что вклад компонент, движущихся в обратном направлении (с отрицательным импульсом), в задаче с начальным значением в некотором смысле эквивалентен вкладу (быстро затухающих) компонент с отрицательной энергией в исходное решение [1]. Таким образом, корректная трактовка некоторых аспектов кинетики волнового пакета (даже в одномерном случае и даже для "свободного" движения) становится нетривиальной задачей и тесно связана с фундаментальной проблемой измерения времен в квантовой механике, таких как время прибытия, время пребывания и время туннелирования (см. [3]).

С другой стороны, связанные с зависимостью от времени аспекты прохождения через потенциальную ступеньку (барьер, яму) и отражения от нее приобрели актуальность и имеют важные практические применения во вновь возникших областях нанонауки и нанотехнологии. Прямоугольные потенциальные барьеры (ямы) часто могут служить удовлетворительным приближением для одномерных потенциальных профилей в слоистых магнитных наноструктурах, в которых имеют место эффекты гигантского магнитосопротивления [4] и туннельного магнитосопротивления [5]. Эти эффекты, которые проистекают, в частности, из квантово-механического рассеяния на потенциальных ямах (барьерах), привели к очень важным коммерческим приложениям. Таким образом, понимание аспектов, связанных с зависимостью от времени в рассеянии на прямоугольном потенциале, необходимо для развития кинетической теории наноструктур.

Решение зависящего от времени уравнения Шредингера можно получить с помощью пространственно-временного пропагатора (функции Грина), который удобно вычислять методом интегралов по траекториям. Список точных решений для такого пропагатора очень невелик. Например, в работе [6] с помощью метода интегралов по траекториям было получено точное решение для пространственно-временного пропагатора для случая одномерного прямоугольного барьера, однако это решение является очень сложным, неявным и с трудом поддается анализу.

Недавно нами был предложен метод [7]-[9] для вычисления пространственно-временного пропагатора, основанный на интегрировании по энергии матрицы спектральной плотности (разрыва зависящей от энергии функции Грина на веществен- 
ной оси энергии). Зависящую от энергии функцию Грина нетрудно при этом получить для потенциальной ступеньки (барьера, ямы) с помощью теории многократного рассеяния, используя найденные в работе [7] эффективные зависящие от энергии потенциалы, ответственные за отражение от потенциальной ступеньки и прохождение через нее. Эти потенциалы, которые определяются через различные скорости частицы по обеим сторонам ступеньчатых потенциалов, составляющих барьер или яму, позволяют рассматривать процессы отражения и прохождения как рассеяние частицы на скачке потенциала, в отличие от общепринятой волновой картины. Важное преимущество нашего подхода к вычислению пропагатора состоит в том, что он допускает естественное разложение эволюции во времени общей исходной волновой функции на компоненты, движущиеся как в прямом $(p=\hbar k>0)$, так и в обратном (с отрицательным импульсом $p<0$ ) направлении, и позволяет анализировать вклады обоих этих слагаемых и их интерференции в отражение и прохождение частицы. Кроме того, использование процедуры с разрешением по энергии автоматически устраняет все нефизические энергетические области, например отрицательные энергии.

В настоящей работе мы применяем наш подход к решению зависящего от времени уравнения Шредингера в случае, когда частица движется в направлении одномерного прямоугольного потенциала, амплитуда которого может изменяться от положительных энергий (яма) до отрицательных (барьер). Получено простое точное решение для зависящего от времени пропагатора в терминах интегралов от элементарных функций, справедливое как для ямы, так и для барьера. Показано, что связанные состояния в яме не дают вклада в решение. Полученный результат дает полное решение зависящего от времени уравнения Шредингера, а также точные аналитические выражения для волновой функции $\psi(x, t)$ в пространственных областях левее, внутри и правее потенциальной ямы (барьера) с учетом слагаемых, соответствующих движению в обратном направлении, обусловленных компонентами с отрицательными импульсами исходной волновой функции. Соответствующие плотности вероятности $|\psi(x, t)|^{2}$ анализируются и численно визуализируются для исходного волнового пакета гауссовой формы, при этом особое внимание уделяется слагаемым, соответствующим движению в обратном направлении, и интерференционным слагаемым. Также получены аналитические выражения для времени прибытия и времени пребывания в различных пространственных областях относительно области потенциальной ямы (барьера). Время пребывания в области потенциальной ямы (барьера) приведено как функция амплитуды потенциала, которая меняется от положительных значений (яма) до отрицательных (барьер).

\section{2. ТОЧНОЕ РЕШЕНИЕ ЗАВИСЯЩЕГО ОТ ВРЕМЕНИ УРАВНЕНИЯ ШРЕДИНГЕРА}

Рассмотрим частицу, движущуюся вдоль оси $x$ по направлению к потенциалу

$$
V(x)=-[\theta(x)-\theta(x-d)] U
$$

соответствующему прямоугольной яме (барьеру), локализованному в области $0<x<d$, где $d>0$ - ширина ямы (барьера), причем $U>0$ - глубина ямы, a $U<0$ - высота барьера. 
Волновую функцию отдельной частицы, движущейся в возмущающем потенциале $V(x)$, можно выразить как [10]

$$
\psi(x, t)=\int d x^{\prime} K\left(x, t ; x^{\prime}, t_{0}\right) \psi\left(x^{\prime}, t_{0}\right) .
$$

Пропагатор $K\left(x, t ; x^{\prime}, t_{0}\right)$ представляет собой амплитуду вероятности перехода частицы из начальной пространственно-временной точки $\left(x^{\prime}, t_{0}\right)$ в конечную точку $(x, t)$ по всем возможным путям. Он дает полную информацию о динамике частицы и решает соответствующее зависящее от времени уравнение Шредингера.

Согласно [7] зависящий от времени запаздывающий пропагатор

$$
K\left(t-t^{\prime}\right)=\theta\left(t-t^{\prime}\right) \exp \left[-\frac{i}{\hbar} H\left(t-t^{\prime}\right)\right],
$$

где $H$ - не зависящий от времени гамильтониан рассматриваемой системы, нетрудно вычислить в пространственном представлении с использованием следующего определения:

$$
K\left(x, t ; x^{\prime}, t_{0}\right)=\theta\left(t-t_{0}\right) \int_{-\infty}^{\infty} \exp \left[-\frac{i}{\hbar} E\left(t-t_{0}\right)\right] A\left(x, x^{\prime} ; E\right) d E .
$$

Здесь $A\left(x, x^{\prime} ; E\right)$ - матрица спектральной плотности

$$
\begin{aligned}
A\left(x, x^{\prime} ; E\right) & =\frac{i}{2 \pi}\left[G^{+}\left(x, x^{\prime} ; E\right)-G^{-}\left(x, x^{\prime} ; E\right)\right], \\
G^{+}\left(x, x^{\prime} ; E\right) & =\left\langle x\left|\frac{1}{E-H+i \varepsilon}\right| x^{\prime}\right\rangle, \quad \varepsilon \rightarrow+0, \\
G^{-}\left(x, x^{\prime} ; E\right) & =\left[G^{+}\left(x^{\prime}, x ; E\right)\right]^{*},
\end{aligned}
$$

определяемая матричными элементами запаздывающей $G^{+}(E)$ и опережающей $G^{-}(E)$ зависящих от энергии операторных функций Грина $G^{ \pm}=(E-H \pm i \varepsilon)^{-1}$, аналитических соответственно в верхней и нижней полуплоскостях комплексной энергии $E$.

В работе [9] мы получили выражения для запаздывающей функции Грина $G^{+}\left(x, x^{\prime} ; E\right)$ для частицы, движущейся из области $x^{\prime}<0$ по направлению к прямоугольному барьеру, локализованному в области $0<x<d$, которые также справедливы для потенциальной ямы, если изменить знак у потенциала $U$, соответствующего высоте барьера, т. е. заменить $U$ на $-U$ (в соответствии с потенциалом (1)). Таким образом, в рассматриваемых пространственных областях имеем

$$
\begin{aligned}
& G^{+}\left(x, x^{\prime} ; E\right)= \\
& = \begin{cases}\frac{m}{i \hbar^{2} k} e^{i k(x-d)} t(E) e^{-i k x^{\prime}}, & x^{\prime}<0, x>d, \\
\frac{m}{i \hbar^{2} k} e^{-i k x} t(E) e^{i k\left(x^{\prime}-d\right)}, & x^{\prime}>d, x<0, \\
\frac{m}{i \hbar^{2} \sqrt{k k_{-u}}}\left[e^{i k_{-u} x} t^{\prime}(E) e^{-i k x^{\prime}}+e^{-i k_{-u} x} r^{\prime}(E) e^{-i k x^{\prime}}\right], & x^{\prime}<0,0<x<d, \\
\frac{m}{i \hbar^{2} \sqrt{k k_{-u}}}\left[e^{-i k x} t^{\prime}(E) e^{i k_{-u} x^{\prime}}+e^{-i k x} r^{\prime}(E) e^{-i k_{-u} x^{\prime}}\right], & x<0,0<x^{\prime}<d, \\
\frac{m}{i \hbar^{2} k}\left[e^{i k\left|x-x^{\prime}\right|}+r(E) e^{-i k\left(x+x^{\prime}\right)}\right], & x<0, x^{\prime}<0,\end{cases}
\end{aligned}
$$


где амплитуды прохождения и отражения определяются как

$$
\begin{aligned}
t(E) & =\frac{4 k k_{-u} e^{i k_{-u} d}}{d(E)}, \quad t^{\prime}(E)=\frac{2 \sqrt{k k_{-u}}\left(k+k_{-u}\right)}{d(E)}, \\
r^{\prime}(E) & =\frac{2 \sqrt{k k_{-u}}\left(k_{-u}-k\right) e^{2 i k_{-u} d}}{d(E)}, \quad r(E)=\frac{\left(k^{2}-k_{-u}^{2}\right)\left(1-e^{2 i k_{-u} d}\right)}{d(E)}, \\
d(E) & =\left(k+k_{-u}\right)^{2}-\left(k-k_{-u}\right)^{2} e^{2 i k_{-u} d} .
\end{aligned}
$$

Волновые числа частицы $k$ и $k_{-u}$ связаны с пространственными областями соответственно снаружи и внутри потенциальной ямы (барьера) и зависят от энергии $E$ следующим образом:

$$
k(E)=\sqrt{\frac{2 m}{\hbar^{2}} E}, \quad k_{-u}(E)=\sqrt{\frac{2 m}{\hbar^{2}}(E+U)} .
$$

Из соотношений (5) мы видим, что $G^{+}\left(x, x^{\prime} ; E\right)=G^{+}\left(x^{\prime}, x ; E\right)$, и поэтому опережающая функция Грина $G^{-}\left(x, x^{\prime} ; E\right)=\left[G^{+}\left(x, x^{\prime} ; E\right)\right]^{*}$ (см. (4)). Таким образом, амплитуду перехода (3), которая определяется мнимой частью функции Грина, можно записать как

$$
K\left(x, t ; x^{\prime}, t_{0}\right)=-\theta\left(t-t_{0}\right) \frac{1}{\pi} \int_{-\infty}^{\infty} d E \exp \left[-\frac{i}{\hbar} E\left(t-t_{0}\right)\right] \operatorname{Im} G^{+}\left(x, x^{\prime} ; E\right)
$$

Формулы (5)-(8) представляют точное решение для пропагатора частицы в потенциале (1) в терминах интегралов от элементарных функций. Следует иметь в виду, что скорость $v(E)=\hbar k(E) / m$, поэтому величины $t(E), t^{\prime}(E), r^{\prime}(E)$ и $r(E)$ в (6) различны для различных областей интегрирования по энергии $-\infty<E<0$ и $0<E<\infty$ : в первом случае $k(E)$ нужно заменить на $i \bar{k}(E)$, где $\bar{k}(E)=\sqrt{2 m|E| / \hbar^{2}}$. Отсюда следует, что “свободная" функция Грина

$$
G_{0}^{+}\left(x, x^{\prime} ; E\right)=\frac{m}{i \hbar^{2} k} e^{i k\left|x-x^{\prime}\right|}
$$

является вещественной в энергетическом интервале $(-\infty \div 0)$, и поэтому на данном интервале она не дает вклада в соответствующий "свободный" пропагатор $K_{0}\left(x, t ; x^{\prime}, t_{0}\right)$, определяемый выражением (8). Следует также заметить, что для отрицательных энергий $E<0$ мнимые части функций Грина (5) обращаются в нуль во всех пространственных областях, как видно из определений (6) и (7) (например, $\operatorname{Im} t(E)=0$ и $\operatorname{Im} r(E)=0$ при $E<0)$. Поэтому энергетический интервал $(-\infty \div 0)$ не дает вклада в распространение частиц (энергия которых, естественно, больше нуля) через область потенциальной ямы (барьера). 
Таким образом, пространственно-временной пропагатор имеет вид

$$
\begin{aligned}
& K\left(x, t ; x^{\prime}, t_{0}\right)=\theta\left(t-t_{0}\right) \frac{1}{\pi \hbar} \int_{0}^{\infty} d E \exp \left[-\frac{i}{\hbar} E\left(t-t_{0}\right)\right] \frac{1}{v(E)} \times \\
& \times \operatorname{Re}\left[t(E) e^{i k\left(x-x^{\prime}-d\right)}\right], \quad x^{\prime}<0, \quad x>d, \\
& K\left(x, t ; x^{\prime}, t_{0}\right)=\theta\left(t-t_{0}\right) \frac{1}{\pi \hbar} \int_{0}^{\infty} d E \exp \left[-\frac{i}{\hbar} E\left(t-t_{0}\right)\right] \frac{1}{\sqrt{v(E)}} \times \\
& \times \operatorname{Re}\left\{\frac{e^{-i k x^{\prime}}}{\sqrt{v_{-u}(E)}}\left[t^{\prime}(E) e^{i k_{-u} x}+r^{\prime}(E) e^{-i k_{-u} x}\right]\right\}, \quad x^{\prime}<0, \quad 0<x<d, \\
& K\left(x, t ; x^{\prime}, t_{0}\right)=\theta\left(t-t_{0}\right) \frac{1}{\pi \hbar} \int_{0}^{\infty} d E \exp \left[-\frac{i}{\hbar} E\left(t-t_{0}\right)\right] \frac{1}{v(E)} \times \\
& \quad \times \operatorname{Re}\left[e^{i k\left|x-x^{\prime}\right|}+r(E) e^{-i k\left(x+x^{\prime}\right)}\right], \quad x^{\prime}<0, \quad x<0,
\end{aligned}
$$

где $v_{-u}(E)=\hbar k_{-u}(E) / m$. Заметим, что соотношения (9) справедливы как для ямы $(U \geqslant 0)$, так и для барьера $(U \leqslant 0)$. Во втором случае скорость $v_{-u}(E)$ и, соответственно, волновое число $k_{-u}(E)$ следует заменить на $v_{u}(E)=\hbar k_{u}(E) / m$, $k_{u}(E)=\sqrt{2 m(E-|U|) / \hbar^{2}}$. В области энергий $0 \leqslant E<|U|$ скорость $v_{u}(E)$ является мнимой и равна $i \bar{v}_{u}(E)=i \hbar \bar{k}_{u}(E) / m, \bar{k}_{u}(E)=\sqrt{2 m(|U|-E) / \hbar^{2}}$. Легко проверить, что интегрирование по $E$ первого слагаемого в последнем соотношении в $(9)$ дает известную формулу для пространственно-временного пропагатора свободно движущейся частицы:

$$
K_{0}\left(x, t ; x^{\prime}, t_{0}\right)=\theta\left(t-t_{0}\right)\left(\frac{m}{2 \pi i \hbar t}\right)^{1 / 2} \exp \left[\frac{i m\left(x-x^{\prime}\right)^{2}}{2 \hbar t}\right], \quad x<0, \quad x^{\prime}<0
$$

Полученные результаты для пропагатора частицы полностью решают (с помощью формулы (2)) зависящее от времени уравнение Шредингера для частицы, движущейся в прямоугольном потенциале (1).

\section{3. ВОЛНОВАЯ ФУНКЦИЯ И ПЛОТНОСТЬ ВЕРОЯТНОСТИ}

Используя формулы (2), (5) и (8), можно представить волновую функцию в различных пространственных областях при $t>t_{0}$ как

$$
\psi(x, t)=\psi_{>}(x, t)+\psi_{<}(x, t) .
$$

Здесь

$$
\begin{aligned}
\psi_{>}(x, t)= & \frac{1}{\sqrt{2 \pi \hbar}} \int_{0}^{\infty} d E \exp \left[-\frac{i}{\hbar} E\left(t-t_{0}\right)\right] \times & \\
& \times \frac{1}{\sqrt{v(E)}} t(E) e^{i k(x-d)} \psi_{>}(E), & x>d,
\end{aligned}
$$




$$
\begin{array}{rlrl}
\psi_{<}(x, t)= & \frac{1}{\sqrt{2 \pi \hbar}} \int_{0}^{\infty} d E \exp \left[-\frac{i}{\hbar} E\left(t-t_{0}\right)\right] \times & & x>d, \\
& \times \frac{1}{\sqrt{v(E)}} t^{*}(E) e^{-i k(x-d)} \psi_{<}(E), & & \\
\psi_{>}(x, t)= & \frac{1}{\sqrt{2 \pi \hbar}} \int_{0}^{\infty} d E \exp \left[-\frac{i}{\hbar} E\left(t-t_{0}\right)\right] \times & & 0<x<d, \\
& \times \frac{1}{\sqrt{v_{-u}(E)}}\left[t^{\prime}(E) e^{i k_{-u} x}+r^{\prime}(E) e^{-i k_{-u} x}\right] \psi_{>}(E), & & \\
\psi_{<}(x, t)= & \frac{1}{\sqrt{2 \pi \hbar}} \int_{0}^{\infty} d E \exp \left[-\frac{i}{\hbar} E\left(t-t_{0}\right)\right] \times x<d, \\
& \times \frac{1}{\sqrt{v_{-u}^{*}(E)}}\left[t^{\prime *}(E) e^{-i k_{-u}^{*} x}+r^{\prime *}(E) e^{i k_{-u}^{*} x}\right] \psi_{<}(E), & & 0<x<0, \\
\psi_{>}(x, t)= & \frac{1}{\sqrt{2 \pi \hbar}} \int_{0}^{\infty} d E \exp \left[-\frac{i}{\hbar} E\left(t-t_{0}\right)\right] \times & & \\
& \times \frac{1}{\sqrt{v(E)}}\left[e^{i k x}+r(E) e^{-i k x}\right] \psi_{>}(E), & & x<0, \\
\frac{1}{\sqrt{2 \pi \hbar}} \int_{0}^{\infty} d E \exp \left[-\frac{i}{\hbar} E\left(t-t_{0}\right)\right] \times & \frac{1}{\sqrt{v(E)}}\left[e^{-i k x}+r^{*}(E) e^{i k x}\right] \psi_{<}(E), &
\end{array}
$$

где, как уже отмечалось, величина скорости $v_{-u}(E)$ может быть мнимой для потенциального барьера $(U<0)$. Исходная волновая функция в $E$-представлении $\psi_{>(<)}(E)$ связана с соответствующей волновой функцией в $k$-представлении $\psi_{>(<)}(k)$ KaK

$$
\psi_{>}(E)=\frac{1}{\sqrt{2 \pi \hbar v(E)}} \psi_{>}[k(E)], \quad \psi_{<}(E)=\frac{1}{\sqrt{2 \pi \hbar v(E)}} \psi_{<}[k(E)]
$$

и

$$
\psi_{>}[k(E)]=\int d x^{\prime} e^{-i k x^{\prime}} \psi\left(x^{\prime}, t_{0}\right), \quad \psi_{<}[k(E)]=\int d x^{\prime} e^{i k x^{\prime}} \psi\left(x^{\prime}, t_{0}\right) .
$$

Можно проверить, что волновые функции (12) и их производные непрерывны при $x=0$ и $x=d$. Для определенности будем предполагать, что при положительных энергиях $k(E)=\sqrt{2 m E / \hbar^{2}}>0$, поэтому $\psi_{>}(k)$ является компонентой исходной волновой функции, соответствующей распространению направо, а $\psi_{<}(k)$ - компонентой, соответствующей распространению налево. Если $U \neq 0$, то интегрирование по $x^{\prime}$ ограничено отрицательным полупространством $\left(x^{\prime}<0\right)$, как следует из выражений (9) для пропагатора частицы.

Результат, выражаемый формулами (11) и (12), указывает, что, вообще говоря, вклад волновой функции, исходно существующей при $t=t_{0}$ слева от потенциала (1) $\left(x^{\prime}<0\right)$, в волновую функцию в области потенциала $(0<x<d)$ и правее $(x>d)$ дается при $t>t_{0}$ компонентами, движущимися как направо $\left(\psi_{>}(k)\right)$, так и налево $\left(\psi_{<}(k)\right)$. Этот довольно парадоксальный результат следует из того факта, что если исходный волновой пакет обладает компонентами с отрицательным импульсом (при ограничении на полупрямую в импульсном пространстве), которыми нельзя пренебречь, то соответствующая пространственная волновая функция отлична от нуля 
во всей области $(-\infty, \infty)$ и, взаимодействуя с барьером даже при $t<t_{0}$, модифицируется этим взаимодействием (см. также [11], [1]). Таким образом, компоненты, движущиеся в обратном направлении, дают вклад в поведение волновой функции при $t>t_{0}$ в пространственных областях справа от области локализации исходного волнового пакета. Соответственно, плотность вероятности $|\psi(x, t)|^{2}$ нахождения частицы в точке пространства-времени $(x, t)$ определяется членами, соответствующими движению в прямом и обратном направлениях, а также интерференционными членами:

$$
|\psi(x, t)|^{2}=\left|\psi_{>}(x, t)\right|^{2}+\left|\psi_{<}(x, t)\right|^{2}+2 \operatorname{Re} \psi_{>}(x, t) \psi_{<}^{*}(x, t) .
$$

Соотношения (12) и (14) можно использовать для численного моделирования плотности вероятности в различных пространственно-временных областях (см. ниже) и для нахождения некоторых характеристик динамики частицы в потенциале (1).

Чтобы оценить вклад слагаемых, соответствующих движению в прямом и обратном направлениях, в полученные общие формулы, нужно рассмотреть физически актуальную ситуацию относительно исходного волнового пакета. Рассмотрим случай, когда движущиеся частицы связаны с волновым пакетом, который исходно достаточно хорошо локализован в области $x^{\prime}<0$. Таким образом, мы теперь рассматриваем задачу в частном случае начального состояния, соответствующего волновому пакету

$$
\psi\left(x^{\prime}, t_{0}\right)=\frac{1}{\left(2 \pi \sigma^{2}\right)^{1 / 4}} \exp \left[-\frac{\left(x^{\prime}-x_{i}\right)^{2}}{4 \sigma^{2}}+i k_{i} x^{\prime}\right], \quad x_{i}<0, \quad k_{i}>0,
$$

локализованному в окрестности $x_{i}<0$ и движущемуся в направлении положительных $x$ со средним импульсом $p_{i}=\hbar k_{i}>0$. Фурье-преобразование функции (15) с учетом (13) дает следующий результат для всего диапазона энергий $(0 \div \infty)$ :

$$
\begin{aligned}
& \psi_{>}(E)=\frac{1}{\sqrt{\pi \hbar v(E)}}\left(2 \pi \sigma^{2}\right)^{1 / 4} \exp \left[i\left[k_{i}-k(E)\right] x_{i}\right] \exp \left[-\left[k_{i}-k(E)\right]^{2} \sigma^{2}\right] \\
& \psi_{<}(E)=\frac{1}{\sqrt{\pi \hbar v(E)}}\left(2 \pi \sigma^{2}\right)^{1 / 4} \exp \left[i\left[k_{i}+k(E)\right] x_{i}\right] \exp \left[-\left[k_{i}+k(E)\right]^{2} \sigma^{2}\right] .
\end{aligned}
$$

Физически актуальная ситуация имеет место, когда исходная волновая функция обращается в нуль при $x>0$ (хорошо локализована в пределах полупрямой $x<0$ ), поскольку пропагатор (9) переводит эту функцию из области $x^{\prime}<0$ в области $x>0$ или $x<0$. Этого можно достичь, если определить исходную волновую функцию в виде $(15)$ при $x^{\prime}<0$ и положить ее равной нулю при $x^{\prime}>0$. Можно показать, что если выполнено условие

$$
\left|\frac{x_{i}}{2 \sigma}\right| \gg 1
$$

(т. е. если хвост исходного волнового пакета (15) очень мал вблизи точки прибытия $x=0)$, то фурье-преобразование исходного волнового пакета совпадает с фурье-преобразованием обрезанного гауссова волнового пакета, определяемого как (15) при $x^{\prime}<0$ и равного нулю при $x^{\prime}>0$ (см. [12]). 
Вообще говоря, как $\psi_{>}(x, t)$-компоненты, так и $\psi_{<}(x, t)$-компоненты дают вклад в плотность вероятности $|\psi(x, t)|^{2}$ (см. (14)). Если также предположить, что

$$
k_{i} \sigma \gg 1
$$

откуда следует, что дисперсия импульса $\hbar / 2 \sigma$ много меньше характеристического импульса $p_{i}=\hbar k_{i}$, или, эквивалентно,

$$
\frac{\hbar^{2}}{2 m \sigma^{2}} \ll E_{i}
$$

т. е. дисперсия энергии $\hbar^{2} / 8 m \sigma^{2}$ много меньше характеристической энергии $E_{i}=$ $\hbar^{2} k_{i}^{2} / 2 m$, то из (12) и (16) можно видеть, что вклад в плотность вероятности члена $\psi_{<}(x, t)$, соответствующего движению в обратном направлении, значительно меньше, чем вклад члена $\psi_{>}(x, t)$, соответствующего движению в прямом направлении, поэтому вкладом $\psi_{<}(x, t)$ можно пренебречь в первом приближении.

Удобно (например, при численной визуализации полученных результатов) перейти к безразмерным переменным. Как видно из (12), имеются естественный пространственный масштаб $d$, энергетический масштаб $E_{d}=\hbar^{2} / 2 m d^{2}$ (энергетическая неопределенность вследствие локализации частицы в области потенциальной ямы (барьера) шириной $d$ ) и соответствующий временной масштаб $t_{d}=\hbar / E_{d}$. Тогда, используя (12) и (16), можно получить волновую функцию в различных пространственных областях из эволюции исходного гауссова волнового пакета (15) в присутствии потенциальной ямы (1). Таким образом, волновая функция (12) в безразмерных переменных имеет вид

$$
\begin{aligned}
& \psi(\tilde{x}, \tilde{t})=\psi_{>}(\tilde{x}, \tilde{t})+\psi_{<}(\tilde{x}, \tilde{t}), \quad \tilde{t}>\tilde{t}_{0}, \\
& \psi_{>}(\tilde{x}, \tilde{t})=\frac{1}{\sqrt{2}(2 \pi)^{3 / 4}} \frac{\tilde{\sigma}^{1 / 2}}{d^{1 / 2}} e^{i k_{i} x_{i}} \times \\
& \times \int_{0}^{\infty} \frac{d \widetilde{E}}{\sqrt{\widetilde{E}}} e^{-i \widetilde{E}\left(\tilde{t}-\tilde{t}_{0}\right)} \tilde{t}(\widetilde{E}) e^{-\left(\sqrt{\widetilde{E}}-\sqrt{\widetilde{E}_{i}}\right)^{2} \tilde{\sigma}^{2}} e^{i \sqrt{\widetilde{E}}\left(\tilde{x}-\tilde{x}_{i}-1\right)}, \quad \tilde{x}>1, \\
& \psi_{<}(\tilde{x}, \tilde{t})=\frac{1}{\sqrt{2}(2 \pi)^{3 / 4}} \frac{\tilde{\sigma}^{1 / 2}}{d^{1 / 2}} e^{i k_{i} x_{i}} \times \\
& \times \int_{0}^{\infty} \frac{d \widetilde{E}}{\sqrt{\widetilde{E}}} e^{-i \widetilde{E}\left(\tilde{t}-\tilde{t}_{0}\right)} \tilde{t}^{*}(\widetilde{E}) e^{-\left(\sqrt{\widetilde{E}}+\sqrt{\widetilde{E}_{i}}\right)^{2} \tilde{\sigma}^{2}} e^{-i \sqrt{\widetilde{E}}\left(\tilde{x}-\tilde{x}_{i}-1\right)}, \quad \tilde{x}>1, \\
& \psi_{>}(\tilde{x}, \tilde{t})=\frac{1}{\sqrt{2}(2 \pi)^{3 / 4}} \frac{\tilde{\sigma}^{1 / 2}}{d^{1 / 2}} e^{i k_{i} x_{i}} \int_{0}^{\infty} \frac{d \widetilde{E}}{\widetilde{E}^{1 / 4}(\widetilde{E}+\widetilde{U})^{1 / 4}} e^{-i \widetilde{E}\left(\tilde{t}-\tilde{t}_{0}\right)}\left[\tilde{t}^{\prime}(\widetilde{E}) e^{i \sqrt{\widetilde{E}+\widetilde{U}} \tilde{x}}+\right. \\
& \left.+\tilde{r}^{\prime}(\widetilde{E}) e^{-i \sqrt{\widetilde{E}+\widetilde{U}} \tilde{x}}\right] e^{-(\sqrt{\widetilde{E}}-\sqrt{\widetilde{E}})^{2} \tilde{\sigma}^{2}} e^{-i \sqrt{\widetilde{E}} \tilde{x}_{i}}, \quad 0<\tilde{x}<1, \\
& \psi_{<}(\tilde{x}, \tilde{t})=\frac{1}{\sqrt{2}(2 \pi)^{3 / 4}} \frac{\tilde{\sigma}^{1 / 2}}{d^{1 / 2}} e^{i k_{i} x_{i}} \int_{0}^{\infty} \frac{d \widetilde{E}}{\widetilde{E}^{1 / 4}\left[(\widetilde{E}+\widetilde{U})^{1 / 4}\right]^{*}} e^{-i \widetilde{E}\left(\tilde{t}-\tilde{t}_{0}\right)} \times \\
& \times\left[\tilde{t}^{\prime *}(\widetilde{E}) e^{-i(\sqrt{\widetilde{E}+\widetilde{U}})^{*} \tilde{x}}+\tilde{r}^{\prime *}(\widetilde{E}) e^{i(\sqrt{\widetilde{E}+\widetilde{U}})^{*} \tilde{x}}\right] e^{-\left(\sqrt{\widetilde{E}}+\sqrt{\widetilde{E}_{i}}\right)^{2} \tilde{\sigma}^{2}} e^{i \sqrt{\widetilde{E}} \tilde{x}_{i}}, \\
& 0<\tilde{x}<1
\end{aligned}
$$




$$
\begin{aligned}
\psi_{>}(\tilde{x}, \tilde{t})= & \frac{1}{\sqrt{2}(2 \pi)^{3 / 4}} \frac{\tilde{\sigma}^{1 / 2}}{d^{1 / 2}} e^{i k_{i} x_{i}} \int_{0}^{\infty} \frac{d \widetilde{E}}{\sqrt{\widetilde{E}}} e^{-i \widetilde{E}\left(\tilde{t}-\tilde{t}_{0}\right)}\left[e^{i \sqrt{\widetilde{E}} \tilde{x}}+\right. \\
& \left.+\tilde{r}(\widetilde{E}) e^{-i \sqrt{\widetilde{E}} \tilde{x}}\right] e^{-(\sqrt{\widetilde{E}}-\sqrt{\widetilde{E}})^{2} \tilde{\sigma}^{2}} e^{-i \sqrt{\widetilde{E}} \tilde{x}_{i}}, \quad \tilde{x}<0, \\
\psi_{<}(\tilde{x}, \tilde{t})= & \frac{1}{\sqrt{2}(2 \pi)^{3 / 4}} \frac{\tilde{\sigma}^{1 / 2}}{d^{1 / 2}} e^{i k_{i} x_{i}} \int_{0}^{\infty} \frac{d \widetilde{E}}{\sqrt{\widetilde{E}}} e^{-i \widetilde{E}\left(\tilde{t}-\tilde{t}_{0}\right)}\left[e^{-i \sqrt{\widetilde{E}} \tilde{x}}+\right. \\
& \left.+\tilde{r}^{*}(\widetilde{E}) e^{i \sqrt{\widetilde{E}} \tilde{x}}\right] e^{-(\sqrt{\widetilde{E}}+\sqrt{\widetilde{E}})^{2} \tilde{\sigma}^{2}} e^{i \sqrt{\widetilde{E}} \tilde{x}_{i}}, \quad \tilde{x}<0,
\end{aligned}
$$

где

$$
\begin{gathered}
\tilde{t}(\widetilde{E})=\frac{4 \sqrt{\widetilde{E}} \sqrt{\widetilde{E}+\widetilde{U}} e^{i \sqrt{\widetilde{E}+\widetilde{U}}}}{\tilde{d}(\widetilde{E})}, \\
\tilde{t}^{\prime}(\widetilde{E})=\frac{2 \widetilde{E}^{1 / 4}(\widetilde{E}+\widetilde{U})^{1 / 4}(\sqrt{\widetilde{E}}+\sqrt{\widetilde{E}+\widetilde{U}})}{\tilde{d}(\widetilde{E})}, \\
\tilde{r}^{\prime}(\widetilde{E})=\frac{2 \widetilde{E}^{1 / 4}(\widetilde{E}+\widetilde{U})^{1 / 4}(\sqrt{\widetilde{E}+\widetilde{U}}-\sqrt{\widetilde{E}}) e^{2 i \sqrt{\widetilde{E}+\widetilde{U}}}}{\tilde{d}(\widetilde{E})}, \\
\tilde{d}(\widetilde{E})=\left(\sqrt{\widetilde{E}}+\sqrt{\widetilde{U}\left(1-e^{2 i} \sqrt{\widetilde{E}+\widetilde{U}}\right)}\right. \\
\tilde{d}(\widetilde{E})
\end{gathered}
$$

и $\widetilde{E}=E / E_{d}, \widetilde{U}=U / E_{d}, \widetilde{E}_{i}=E_{i} / E_{d}, E_{i}=\hbar^{2} k_{i}^{2} / 2 m, \tilde{t}=t / t_{d}, \tilde{t}_{0}=t_{0} / t_{d}, \tilde{\sigma}=\sigma / d$, $\tilde{x}=x / d, \tilde{x}_{i}=x_{i} / d$. Условия (17) и (19), записанные в безразмерных переменных, соответственно имеют вид

$$
\left|\tilde{x}_{i}\right| \gg 2 \tilde{\sigma}, \quad \widetilde{E}_{i} \gg \frac{1}{\tilde{\sigma}^{2}}
$$

Поучительно рассмотреть сначала предельный случай, определяемый вторым неравенством $(22)$. В этом случае члены $\psi_{>}(\tilde{x}, \tilde{t})$, соответствующие движению в прямом направлении в формулах $(20)$, дают основной вклад в полную волновую функцию, т. е. $\psi(\tilde{x}, \tilde{t}) \approx \psi_{>}(\tilde{x}, \tilde{t})$. Кроме того, интегралы по энергии в $\psi_{>}(\tilde{x}, \tilde{t})(20)$ можно асимптотически вычислить при $\widetilde{E}_{i} \widetilde{\sigma}^{2} \gg 1$ в силу того, что основной вклад в эти интегралы главный образом происходит из области энергии $\widetilde{E} \approx \widetilde{E}_{i}$. В этом случае волновые функции $\psi_{>}(\tilde{x}, \tilde{t})$ сводятся (в первом приближении по $1 / \widetilde{E}_{i} \tilde{\sigma}^{2} \ll 1$ ) к стационарным (при $\widetilde{E}=\widetilde{E}_{i}$ ) результатам, осциллирующим во времени по закону $e^{-i \widetilde{E}_{i}\left(\tilde{t}-\tilde{t}_{0}\right)}$. Таким образом, если представить соотношения $(20)$ для $\psi_{>}(\tilde{x}, \tilde{t})$ как

$$
\begin{aligned}
& \psi_{>}(\tilde{x}, \tilde{t})=\int_{0}^{\infty} \varphi\left(\tilde{x}, \tilde{x}_{i} ; \widetilde{E}\right) e^{-i \widetilde{E}\left(\tilde{t}-\tilde{t}_{0}\right)} e^{\lambda f(\widetilde{E})} d \widetilde{E} \\
& \lambda=\widetilde{E}_{i} \widetilde{\sigma}^{2} \gg 1, \quad f(\widetilde{E})=-\frac{\left(\sqrt{\widetilde{E}}-\sqrt{\widetilde{E}_{i}}\right)^{2}}{\widetilde{E}_{i}},
\end{aligned}
$$


где $\varphi\left(\tilde{x}, \tilde{x}_{i} ; \widetilde{E}\right)$ обозначает любое подынтегральное выражение из $(20)$, умноженное на экспоненты из (23), то асимптотическое значение выражения (23) будет равно

$$
\psi_{>}(\tilde{x}, \tilde{t})=\frac{2 \sqrt{\pi}}{\tilde{\sigma}} \sqrt{\widetilde{E}_{i}} \varphi\left(\tilde{x}, \tilde{x}_{i} ; \widetilde{E}_{i}\right) e^{-i \widetilde{E}_{i}\left(\tilde{t}-\tilde{t}_{0}\right)} .
$$

Соответственно, квадрат модуля $\left|\psi_{>}(\tilde{x}, \tilde{t})\right|^{2}$ волновых функций, определяемых формулами (20) и (21), в этом асимптотическом случае не зависит от времени. Как в случае потенциальной ямы $(U>0)$, так и в случае потенциального барьера при $E_{i}>|U|(U<0)$ имеем (в исходных немасштабированных переменных)

$$
\begin{aligned}
& \left|\psi_{>}(x, t)\right|^{2}= \\
& =\left\{\begin{array}{l}
\frac{1}{\sqrt{2 \pi} \sigma} \frac{16 E_{i}\left(E_{i} \pm|U|\right)}{\left|d\left(E_{i}\right)\right|^{2}}, \quad x>d, \\
\frac{16}{\sqrt{2 \pi} \sigma} \frac{E_{i}}{\left|d\left(E_{i}\right)\right|^{2}}\left\{E_{i} \pm|U| \cos ^{2}\left[\sqrt{\frac{2 m\left(E_{i} \pm|U|\right)}{\hbar^{2}}}(x-d)\right]\right\}, \quad 0<x<d, \\
\frac{1}{\sqrt{2 \pi} \sigma}\left\{1+\frac{4 U^{2} \sin ^{2}\left(\sqrt{2 m\left(E_{i} \pm|U|\right) / \hbar^{2}} d\right)}{\left|d\left(E_{i}\right)\right|^{2}} \pm \frac{8|U|}{\left|d\left(E_{i}\right)\right|^{2}} \times\right. \\
\times\left[2 \sqrt{E_{i}} \sqrt{E_{i}} \pm|U| \sin \left(2 \sqrt{\frac{2 m\left(E_{i} \pm|U|\right)}{\hbar^{2}}} d\right) \sin \left(2 \sqrt{\frac{2 m E_{i}}{\hbar^{2}}} x\right)-\right. \\
-\left(2 E_{i} \pm|U|\right) \sin ^{2}\left(\sqrt{\left.\left.\left.\frac{2 m\left(E_{i} \pm|U|\right)}{\hbar^{2}} d\right) \cos \left(2 \sqrt{\frac{2 m E_{i}}{\hbar^{2}}} x\right)\right]\right\}, \quad x<0},\right. \\
\left|d\left(E_{i}\right)\right|^{2}=16 E_{i}\left(E_{i} \pm|U|\right)+4 U^{2} \sin ^{2}\left[\sqrt{\frac{2 m\left(E_{i} \pm|U|\right)}{\hbar^{2}}} d\right], \quad E_{i} \gg \frac{\hbar^{2}}{2 m \sigma^{2}} .
\end{array}\right.
\end{aligned}
$$

Здесь выбор знака плюс соответствует потенциальной яме, а знака минус - потенциальному барьеру. Формулы (25) определяют пространственную зависимость квадрата модуля волновой функции в различных пространственных областях по отношению к области потенциала в стационарном случае, когда для исходного волнового пакета (15) характерно особенно узкое распределение в энергетическом (импульсном) пространстве. Например, в данном приближении перенесенная сквозь яму (барьер) плотность вероятности $(x>d)$ постоянна в пространстве, в то время как интерференционная картина в области потенциальной ямы (барьера) $(0<x<d)$ представляет собой зависимость типа квадрата косинуса, а в области левее потенциальной ямы (барьера) $(x<0)$ картина более сложная и представляет собой результат интерференции падающих и отраженных волн. Для резонансных состояний, когда $\sqrt{2 m\left(E_{i} \pm|U|\right) / \hbar^{2}} d=\pi n$ ( $n$ - целое число), соотношения (25) упрощаются: слагаемые, обусловленные отражением в область $x<0$, обращаются в нуль, т. е. имеется только плотность вероятности $1 / \sqrt{2 \pi} \sigma$, соответствующая падающей волне, и, соответственно, эта плотность вероятности полностью проходит в область $x>d$.

Зависимость $|\psi(x, t)|^{2}$ от времени проявляется, только если имеется достаточная дисперсия импульса, как следует из формул (20). Пространственно-временную зависимость плотности вероятности можно визуализовать путем численного моделирования. Сосредоточимся на плотности вероятности $|\psi(\tilde{x}, \tilde{t})|^{2}$ нахождения частицы в точке масштабированного пространства-времени $(\tilde{x}, \tilde{t})$ внутри потенциальной ямы 


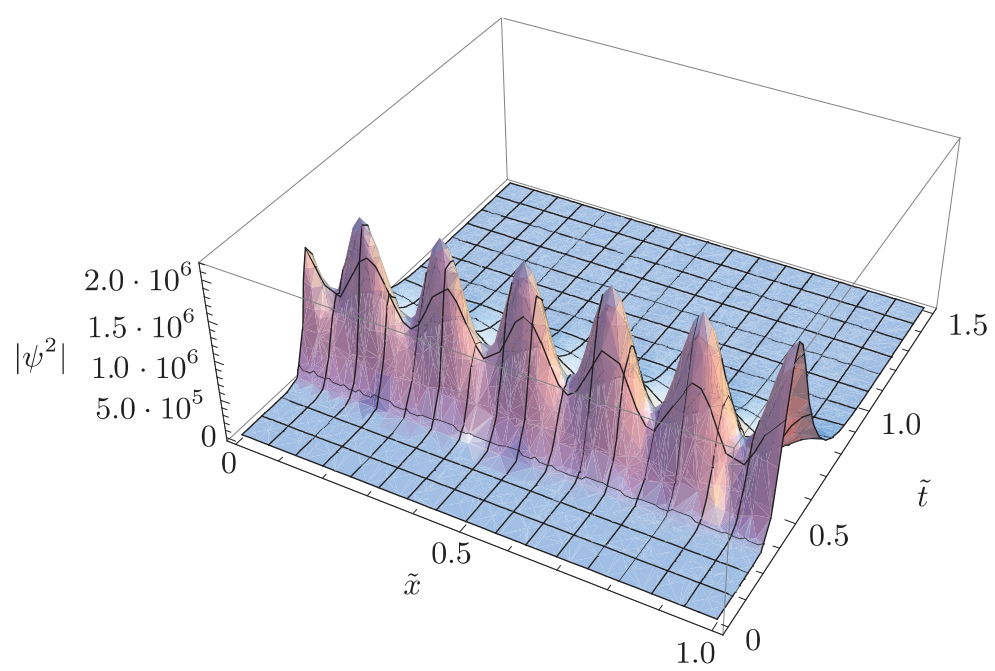

Рис. 1. Распределение плотности вероятности $|\psi(\tilde{x}, \tilde{t})|^{2}$ внутри области потенциальной ямы для узкого распределения по энергиям исходного волнового пакета $(\tilde{\sigma}=1 / 3)$.

в области $\tilde{x}=0 \div 1$ и построим ее график, предвосхищая рассмотрение в следующем разделе, касающееся времени пребывания частицы в данной области. Чтобы сделать динамику волнового пакета больше похожей на динамику частицы, будем считать волновой пакет узким, $\widetilde{\sigma}<1$, и положим $\tilde{t}_{0}=0$. Для электрона и ямы шириной $d=10^{-7}$ см (1 нм) характерная энергия $E_{d} \sim 3 \cdot 10^{-2}$ эВ, а характерное время $t_{d} \sim 2 \cdot 10^{-14}$ с. В соответствии с принятыми условиями положим $\tilde{\sigma}=1 / 3$ и $\tilde{\sigma}=0.05$, $\tilde{x}_{i}=-10, \widetilde{E}_{i}=10^{2}$ и $\widetilde{U}=2 \widetilde{E}_{i}$. Сравним два случая: $\tilde{\sigma}=1 / 3$, когда второе неравенство (22) выполнено и компоненты исходного волнового пакета с положительной энергией, движущиеся в обратном направлении, несущественны, и $\tilde{\sigma}=0.05$, когда их вклад имеет значение. Безразмерный временной интервал $\tilde{t}=0.1 \div 1.5$ выбран из простой оценки для среднего масштабированного времени $t_{i} / t_{d}$, которое требуется частице с начальной энергией $\widetilde{E}_{i}=10^{2}$, чтобы достичь потенциала, если она стартует из точки $\tilde{x}_{i}=-10$ :

$$
\frac{t_{i}}{t_{d}}=\frac{\left|x_{i}\right| m}{\hbar k_{i} t_{d}}=\frac{\left|\tilde{x}_{i}\right|}{2 \sqrt{\widetilde{E}_{i}}}=\frac{1}{2} .
$$

Поведение плотности вероятности $|\psi(\tilde{x}, \tilde{t})|^{2}$ в пространстве и во времени внутри области потенциальной ямы $\tilde{x}=0 \div 1$, определяемое соотношениями $(20)$, показано на рис. 1 и рис. 2. На рис. $1(\tilde{\sigma}=1 / 3)$ показана интерференционная картина ожидаемая зависимость типа квадрата косинуса в фиксированный момент времени (см. (25)). Однако амплитуда этой интерференционной картины увеличивается со временем от нуля до максимального значения (которого она достигает приблизительно при $\tilde{t}=0.5)$, после чего она снова уменьшается до нуля, тем самым демонстрируя конечное время, в течение которого частица существует в области потенциальной ямы до того, как покинуть ее и переместиться в область левее $(\tilde{x}<0)$ или 


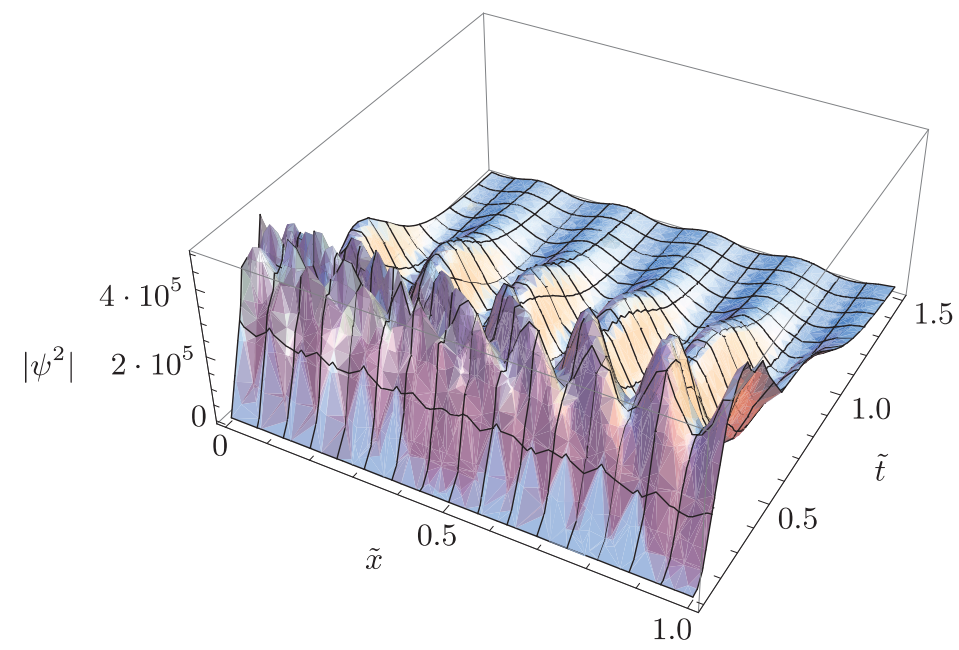

Рис. 2. Распределение плотности вероятности $|\psi(\tilde{x}, \tilde{t})|^{2}$ внутри области потенциальной ямы для широкого распределения по энергиям исходного волнового пакета $(\tilde{\sigma}=0.05)$.

правее $(\tilde{x}>1)$ ямы. На рис. $2(\tilde{\sigma}=0.05)$ видно, что интерференционная картина $|\psi(\tilde{x}, \tilde{t})|^{2}$ является более структурированной в пространстве и во времени. В частности, распределение плотности вероятности во времени является немонотонным и более широким (что указывает на большую величину времени пребывания) по сравнению с предыдущим случаем. Эти изменения в распределении плотности вероятности обусловлены влиянием компонент волновой функции $\psi_{<}(\tilde{x}, \tilde{t})$, движущихся в обратном направлении.

\section{4. ВРЕМЯ ПРИБЫТИЯ И ВРЕМЯ ПРЕБЫВАНИЯ}

Формулы (12) и (14) можно использовать для нахождения некоторых характеристик динамики частицы в потенциале (1). Например, можно определить полную вероятность прибытия частицы в пространственную область правее ямы (барьера) $x>d$ и в пространственную область левее ямы (барьера) $x<0$ как (см. [13], [8])

$$
\begin{aligned}
& P_{>}(\infty)=\lim _{t-t_{0} \rightarrow \infty} \lim _{\varepsilon \rightarrow+0} \int_{d}^{\infty} e^{-\varepsilon x}|\psi(x, t)|^{2} d x, \\
& P_{<}(\infty)=\lim _{t-t_{0} \rightarrow \infty} \lim _{\varepsilon \rightarrow+0} \int_{-\infty}^{0} e^{\varepsilon x}|\psi(x, t)|^{2} d x .
\end{aligned}
$$

Учитывая соотношения (12) для соответствующих пространственных областей (первая, вторая, пятая и шестая формулы в (12)) и интегрируя по комплексной энергетической плоскости (или используя теорему Абеля), получаем

$$
\begin{aligned}
& P_{>}(\infty)=\int_{0}^{\infty}|t(E)|^{2}\left|\psi_{>}(E)\right|^{2} d E \\
& P_{<}(\infty)=\int_{0}^{\infty}\left|r(E) \psi_{>}(E)+\psi_{<}(E)\right|^{2} d E .
\end{aligned}
$$


Видно, что хотя члены, соответствующие движению в обратном направлении, и интерференционные члены в общем случае дают вклад в плотность вероятности (14), они не дают вклада в асимптотику времени прибытия (27) в область правее ямы (барьера) и дают вклад в область слева от ямы (барьера). Поэтому парадоксальный вклад компонент, движущихся в обратном направлении, во время прибытия в область $x>d$ представляет собой переходный во времени эффект, который асимптотически исчезает. В отсутствие потенциала $(U=0),|t(E)|^{2}=1, r(E)=0$ (см. (6)), соотношения (27) сводятся к естественным результатам для свободного движения (см. также работы [14] и [8], где обсуждаются "идеальное" время прибытия (26) и связь между членами, соответствующими движению в обратном направлении, и вкладом, обусловленным отрицательными энергиями). Нетрудно показать, что для конечного пространственного интервала прибытия из определения (26) естественно получается нулевой результат, поскольку частица покидает данную конечную область за достаточно большой интервал времени $\left(t-t_{0} \rightarrow \infty\right)$.

Для конечного пространственного интервала традиционно используется так называемое время пребывания, т. е. среднее время, потраченное в данном интервале частицей, описываемой пакетом $\psi(x, t)$. Время пребывания в области потенциала $(0, d)$ определяется как

$$
\tau(0, d)=\lim _{t-t_{0} \rightarrow \infty} \int_{t_{0}}^{t} d t \int_{0}^{d} d x|\psi(x, t)|^{2} .
$$

Подставляя третье и четвертое соотношения из (12) в определение (28), получаем

$$
\begin{aligned}
\tau(0, d) & =\tau_{>}(0, d)+\tau_{<}(0, d)+2 \operatorname{Re} \tau_{><}(0, d), \\
\tau_{>}(0, d) & =\int_{0}^{\infty} d E \int_{0}^{d} d x\left|\frac{\varphi(x, E)}{\sqrt{v_{-u}(E)}} \psi_{>}(E)\right|^{2}, \\
\tau_{<}(0, d) & =\int_{0}^{\infty} d E \int_{0}^{d} d x\left|\frac{\varphi(x, E)}{\sqrt{v_{-u}(E)}} \psi_{<}(E)\right|^{2}, \\
\tau_{><}(0, d) & =\int_{0}^{\infty} d E \int_{0}^{d} d x\left[\frac{\varphi(x, E)}{\sqrt{v_{-u}(E)}}\right]^{2} \psi_{>}(E) \psi_{<}^{*}(E), \\
\varphi(x, E) & =t^{\prime}(E) e^{i k_{-u} x}+r^{\prime}(E) e^{-i k_{-u} x} .
\end{aligned}
$$

Мы снова видим, что время пребывания определяется компонентами исходного волнового пакета, движущимися в прямом и обратном направлениях, а также их интерференцией. Весь диапазон энергий $(0 \div \infty)$ дает вклад во время пребывания. Из (29) и (12) нетрудно получить, что составляющие времени пребывания, соответствующие движению в прямом и обратном направлениях, равны

$$
\tau_{>(<)}(0, d)=\int_{0}^{\infty} d E \tau_{>(<)}(E ; d) .
$$

Зависящее от энергии время пребывания на единичный интервал энергий $\tau_{>(<)}(E ; d)$, обусловленное компонентами исходного волнового пакета, движущимися в прямом 
(обратном) направлении $\psi_{>(<)}(E)$, имеет вид

$$
\begin{gathered}
\tau_{>(<)}(E ; d)=t(E ; d)\left|\psi_{>(<)}(E)\right|^{2}, \\
t(E ; d)=\frac{k}{v_{-u}} \frac{2 k_{-u} d\left(k_{-u}^{2}+k^{2}\right)+k_{0}^{2} \sin \left(2 k_{-u} d\right)}{4 k^{2} k_{-u}^{2}+k_{0}^{4} \sin ^{2}\left(k_{-u} d\right)},
\end{gathered}
$$

где $k_{0}^{2}=k_{-u}^{2}-k^{2}=\left(2 m / \hbar^{2}\right) U$. Заметим, что $\psi_{>(<)}(E)$ имеет размерность обратного квадратного корня из энергии (см. (16)), поэтому выражение для $t(E ; d)$ имеет размерность времени и совпадает с зависящим от энергии временем пребывания, полученным ранее в работе [15].

Как следует из (29) и (12), обусловленное интерференцией время пребывания на единичный интервал энергий можно записать как

$$
\begin{aligned}
& \tau_{><}(E ; d)=\operatorname{Re}\left\{\frac{4 k}{v_{-u}} \times\right. \\
& \left.\times \frac{\left(k^{2}+k_{-u}^{2}\right) \sin \left(2 k_{-u} d\right)+2 k_{-u} k_{0}^{2} d-4 i k k_{-u} \sin ^{2}\left(k_{-u} d\right)}{\left[i\left(k^{2}+k_{-u}^{2}\right) \sin \left(k_{-u} d\right)-2 k k_{-u} \cos \left(k_{-u} d\right)\right]^{2}} \psi_{>}(E) \psi_{<}^{*}(E)\right\}, \\
& 2 \operatorname{Re} \tau_{><}(0, d)=\int_{0}^{\infty} d E \tau_{><}(E ; d) \text {. }
\end{aligned}
$$

Заметим, что и соотношения (31), и соотношения (32) справедливы как для ямы $(U>0)$, так и для барьера $(U<0)$. Во втором случае нужно выполнить подстановку $U \rightarrow-U\left(k_{-u} \rightarrow k_{u}=\sqrt{\left(2 m / \hbar^{2}\right)(E-|U|)}\right)$, а интегрирование по энергии $0<E<\infty$ включает область $E<|U|$, в которой $k_{u}$ заменяется на $i \bar{k}_{u}, \bar{k}_{u}=\sqrt{\left(2 m / \hbar^{2}\right)(|U|-E)}$. Таким образом, полученные результаты можно использовать для вычисления времени пребывания как для прямоугольной ямы, так и для прямоугольного барьера, а также для рассмотрения его эволюции, когда яма преобразуется в барьер $(U \rightarrow-U)$.

Таким образом, полное время пребывания на единичную энергию

$$
\begin{gathered}
\tau(E ; d)=t(E ; d)\left[\left|\psi_{>}(E)\right|^{2}+\left|\psi_{<}(E)\right|^{2}\right]+\tau_{><}(E ; d), \\
\tau(0, d)=\int_{0}^{\infty} \tau(E ; d) d E
\end{gathered}
$$

в общем случае определяется компонентами исходного волнового пакета, движущимися как в прямом, так и в обратном направлении, а также их интерференцией. Для резонансных энергий, удовлетворяющих условию $k_{-u} d=\pi n(n-$ целое число, $k_{-u}$ - вещественное число), которые имеют место в случаях $U>0$ и $U<0$ (однако только при $E>|U|$ ) и для которых $t(E)= \pm 1, r(E)=0$ (см. (6)), формула (33) принимает вид

$$
\tau(E ; d)=\frac{d}{v}\left\{\frac{1}{2} \frac{k_{-u}^{2}+k^{2}}{k_{-u}^{2}}\left[\left|\psi_{>}(E)\right|^{2}+\left|\psi_{<}(E)\right|^{2}\right]+\frac{k_{0}^{2}}{k_{-u}^{2}} 2 \operatorname{Re}\left[\psi_{>}(E) \psi_{<}^{*}(E)\right]\right\},
$$

где $d / v(E)$ - время, за которое частица с энергией $E$ проходит пространственную область размера $d$ в отсутствие потенциала. Таким образом, выражение в фигурных скобках в (34) показывает разницу между временем пребывания в области потенциальной ямы (барьера) и “свободным" временем пребывания $d / v(E)$. 
Из сказанного выше следует, что в общем случае время пребывания зависит энергетического спектра исходного волнового пакета $\psi_{>(<)}(E)$ и его нереально определить, например, просто через $t(E ; d)(31)$ (то же самое, разумеется, справедливо для времени прибытия). Интересно рассмотреть энергетическую зависимость времени пребывания (33) и влияние на него компонент исходного волнового пакета $\psi_{<}(E)$, движущихся в обратном направлении. Для этого используем гауссов исходный волновой пакет, задаваемый формулами (15) и (16), и перейдем к безразмерным переменным, определенным в предыдущем разделе. В результате из (30)-(32) получаем

$$
\begin{aligned}
& \tau(0, d)=\int_{0}^{\infty} d \widetilde{E} \tilde{\tau}(\widetilde{E} ; d), \quad \tilde{\tau}(\widetilde{E} ; d)=\tilde{\tau}_{>}(\widetilde{E} ; d)+\tilde{\tau}_{<}(\widetilde{E} ; d)+\tilde{\tau}_{><}(\widetilde{E} ; d), \\
& \tilde{\tau}_{>}(\widetilde{E} ; d)+\tilde{\tau}_{<}(\widetilde{E} ; d)=\frac{t_{d} \tilde{\sigma}}{2 \sqrt{2 \pi}} \frac{1}{\sqrt{\widetilde{E}+\widetilde{U}}} \frac{2 \sqrt{\widetilde{E}+\widetilde{U}}(2 \widetilde{E}+\widetilde{U})+\widetilde{U} \sin (2 \sqrt{\widetilde{E}+\widetilde{U}})}{4 \widetilde{E}(\widetilde{E}+\widetilde{U})+\widetilde{U}^{2} \sin ^{2}(\sqrt{\widetilde{E}+\widetilde{U}})} \times \\
& \quad \times\left\{\exp \left[-2\left(\sqrt{\widetilde{E}_{i}}-\sqrt{\widetilde{E}}\right)^{2} \tilde{\sigma}^{2}\right]+\exp \left[-2\left(\sqrt{\widetilde{E}_{i}}+\sqrt{\widetilde{E}}\right)^{2} \tilde{\sigma}^{2}\right]\right\}, \\
& \tilde{\tau}_{><}(\widetilde{E} ; d)=\frac{2 t_{d} \tilde{\sigma}}{\sqrt{2 \pi}} \times \operatorname{Re}\left\{\frac{e^{-2 i \sqrt{\widetilde{E}}} \tilde{x}_{i}}{\sqrt{\widetilde{E}+\widetilde{U}} \times}\right. \\
& \quad \times \frac{(2 \widetilde{E}+\widetilde{U}) \sin (2 \sqrt{\widetilde{E}+\widetilde{U}})+2 \sqrt{\widetilde{E}+\widetilde{U}} \widetilde{U}-4 i \sqrt{\widetilde{E}} \sqrt{\widetilde{E}+\widetilde{U}} \sin ^{2}(\sqrt{\widetilde{E}+\widetilde{U}})}{[i(2 \widetilde{E}+\widetilde{U}) \sin (\sqrt{\widetilde{E}+\widetilde{U}})-2 \sqrt{\widetilde{E}} \sqrt{\widetilde{E}+\widetilde{U}} \cos (\sqrt{\widetilde{E}+\widetilde{U}})]^{2}} \times \\
& \quad \times \exp \left[-(\sqrt{\widetilde{E}}+\sqrt{\widetilde{E}})^{2} \tilde{\sigma}^{2}\right] \exp \left[-(\sqrt{\widetilde{E}}+\sqrt{\widetilde{E}})^{2} \tilde{\sigma}^{2}\right],
\end{aligned}
$$

где характеристическое время

$$
\frac{t_{d}}{2}=\frac{\hbar}{2 E_{d}}=\frac{d}{v(E)} \sqrt{\widetilde{E}}=\frac{d}{v\left(E_{i}\right)} \sqrt{\widetilde{E}_{i}},
$$

т. е. оно равно времени, проведенному “свободной" частицей с энергией $E=E_{d}$ $(\widetilde{E}=1)$ в области потенциальной ямы (барьера) шириной $d$, поэтому $\tilde{\tau}(\widetilde{E} ; d)$ имеет размерность времени. Относительные вклады компонент $\tilde{\tau}_{>(<)}(\widetilde{E} ; d)$, движущихся в прямом (обратном) направлении, и интерференционного члена $\tilde{\tau}_{><}(\widetilde{E} ; d)$ во время пребывания $\tau(0, d)(35)$ зависят от значения параметра $\widetilde{E}_{i} \widetilde{\sigma}^{2}$. Если выполнено второе неравенство $(22)$, т. е. $\widetilde{E}_{i} \tilde{\sigma}^{2} \gg 1$, то вклады во время пребывания (35) компонент, движущихся в обратном направлении, и интерференционных слагаемых много меньше, чем вклады компонент $\widetilde{\tau}_{>}(\widetilde{E} ; d)$, движущихся в прямом направлении, поэтому в первом приближении в пределе, задаваемом условиями (22), членами первого типа можно пренебречь. Более того, интеграл от $\tilde{\tau}_{>}(\widetilde{E} ; d)$ по $\widetilde{E}$ можно оценить асимптотически, поскольку при $\widetilde{E}=\widetilde{E}_{i}$ подынтегральное выражение имеет острый максимум. В результате получим

$$
\tau(0, d) \sim \tau_{>}(0, d)=\frac{d}{v\left(E_{i}\right)} \frac{\widetilde{E}_{i}}{\sqrt{\widetilde{E}_{i}+\widetilde{U}}} \frac{2 \sqrt{\widetilde{E}_{i}+\widetilde{U}}\left(2 \widetilde{E}_{i}+\widetilde{U}\right)+\widetilde{U} \sin \left(2 \sqrt{\widetilde{E}_{i}+\widetilde{U}}\right)}{4 \widetilde{E}_{i}\left(\widetilde{E}_{i}+\widetilde{U}\right)+\widetilde{U}^{2} \sin ^{2}\left(\sqrt{\widetilde{E}_{i}+\widetilde{U}}\right)},
$$

что совпадает с выражением (31) для $t\left(E_{i} ; d\right)$, записанным в безразмерных переменных. Следует подчеркнуть, что этот результат представляет только первый член 
асимптотического разложения выражения $\int_{0}^{\infty} d \widetilde{E} \tau_{>}(\widetilde{E} ; d)$ при малом значении параметра $1 / \widetilde{E}_{i} \tilde{\sigma}^{2}$, т. е. для исходного волнового пакета с узким распределением импульсов.

Для резонансных энергий, удовлетворяющих условию $k_{-u} d=\pi n(n-$ целое число, отличное от нуля, $k_{-u}$ - вещественное число), которое в безразмерных переменных записывается как $\widetilde{E}_{i}+\widetilde{U}=\pi^{2} n^{2}$, выражение (36) принимает вид

$$
\frac{\tau_{>}^{r}(0, d) v\left(E_{i}\right)}{d}=\frac{2 \widetilde{E}_{i}+\widetilde{U}}{2\left(\widetilde{E}_{i}+\widetilde{U}\right)}=\frac{\widetilde{E}_{i}+\pi^{2} n^{2}}{2 \pi^{2} n^{2}} .
$$

При $\widetilde{U}>0$ (яма) должно удовлетворяться неравенство $\widetilde{U}=\pi^{2} n^{2}-\widetilde{E}_{i}>0$ ( $n$ ограничено снизу), и при $\pi^{2} n^{2} \gg \widetilde{E}_{i}$, что имеет место при достаточно больших $\widetilde{U}$, асимптотическое относительное резонансное время пребывания (37) стремится к $1 / 2$. При $\widetilde{U}<0$ (барьер) должно удовлетворяться условие $|\widetilde{U}|=\widetilde{E}_{i}-\pi^{2} n^{2}>0\left(\widetilde{E}_{i}>|\widetilde{U}|\right.$, $n$ ограничено малыми значениями, определяемыми $\left.\widetilde{E}_{i}\right)$, и при $\pi^{2} n^{2} \ll \widetilde{E}_{i}$ время пребывания (37) изменяется как $\widetilde{E}_{i} / 2 \pi^{2} n^{2}$.

Если $\widetilde{E}_{i}+\widetilde{U} \rightarrow 0$ (точки поворота в классической физике), что может иметь место только при $\widetilde{U}<0$, асимптотическое относительное время пребывания $\tau_{>}(0, d) v\left(E_{i}\right) / d$ (36) сводится к

$$
\frac{\tau_{>}(0, d) v\left(E_{i}\right)}{d}=\frac{4 \widetilde{E}_{i}}{4 \widetilde{E}_{i}+\widetilde{U}^{2}}<1, \quad \widetilde{E}_{i}-|\widetilde{U}| \rightarrow 0
$$

В отсутствие потенциала $(U=0)$ время пребывания равно "свободному" времени пребывания $d / v\left(E_{i}\right)$.

Интересно рассмотреть график зависимости относительного времени пребывания (36) от амплитуды потенциала $\widetilde{U}$, меняющейся от положительных значений (яма) до отрицательных (барьер). Формула (36) справедлива как для $\widetilde{U}>0$, так и для $\widetilde{U}<0$, причем во втором случае при $\widetilde{E}_{i}-|\widetilde{U}|<0$ соотношение (31) принимает вид

$$
\frac{\tau_{>}(0, d) v\left(E_{i}\right)}{d}=\frac{\widetilde{E}_{i}}{\sqrt{|\widetilde{U}|-\widetilde{E}_{i}}} \frac{2 \sqrt{|\widetilde{U}|-\widetilde{E}_{i}}\left(|\widetilde{U}|-2 \widetilde{E}_{i}\right)+|\widetilde{U}| \operatorname{sh}\left(2 \sqrt{|\widetilde{U}|-\widetilde{E}_{i}}\right)}{4 \widetilde{E}_{i}\left(|\widetilde{U}|-\widetilde{E}_{i}\right)+\widetilde{U}^{2} \operatorname{sh}^{2}\left(\sqrt{|\widetilde{U}|-\widetilde{E}_{i}}\right)}
$$

На рис. 3 показана зависимость $\tau_{>}(0, d) v\left(E_{i}\right) / d(36)$ от $\widetilde{U}$ для широкого диапазона изменения $\widetilde{U}=-5 \cdot 10^{2} \div 10^{3}$ и $\widetilde{E}_{i}=10^{2}$. Можно видеть последовательность резонансов при $\widetilde{U}>0$, амплитуды которых стремятся к $1 / 2$ при достаточно больших $n$, $n^{2} \gg \widetilde{E}_{i} / \pi^{2}$, в то время как при $\widetilde{U}<0$ имеется ограниченная последовательность резонансов с $n^{2}<\widetilde{E}_{i} / \pi^{2}$ (для $\widetilde{E}_{i}>|\widetilde{U}|$ ) с бо́льшими амплитудами, поскольку в области потенциального барьера частица движется медленнее, чем в области потенциальной ямы. Такое поведение соответствует приведенному выше анализу. 


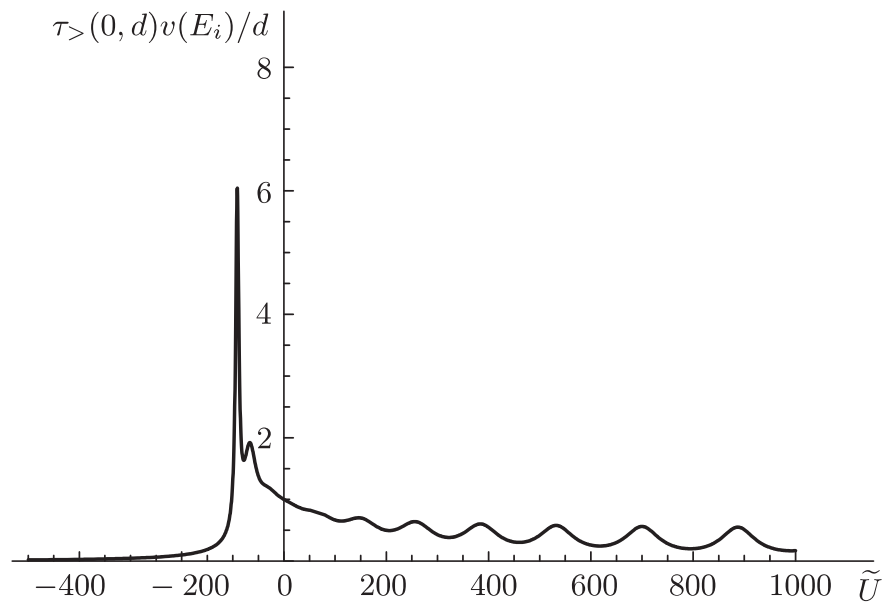

Рис. 3. Зависимость асимптотического времени пребывания от амплитуды потенциальной ямы (барьера) для сверхузкого распределения по энергии исходного волнового пакета $\left(\widetilde{E}_{i} \tilde{\sigma}^{2} \rightarrow \infty\right)$.

Для более реалистичной оценки времени $\tau(0, d)$, задаваемого в $(35)$, и влияния на него компонент начальной волновой функции численно проинтегрируем по $\widetilde{E}$ безразмерное время пребывания $\tau(0, d) v\left(E_{i}\right) / d=2 \sqrt{\widetilde{E}_{i}} \tau(0, d) / t_{d}$ при $\widetilde{E}_{i}=10^{2}$ и $\widetilde{U}=-200 \div 10^{3}$. Заметим, что выражение $(35)$ имеет особенность при $\widetilde{E}=0$ в отсутствие потенциала $(\widetilde{U}=0)$. Чтобы избежать этой особенности, исключим точку $\widetilde{U}=0$ из области изменения $\widetilde{\widetilde{U}}$. На рис. 4 показана зависимость от $\widetilde{U}$ безразмерного времени пребывания при $\tilde{\sigma}=1 / 3$, т. е. когда вклад слагаемых $\tilde{\tau}_{<}(\widetilde{E} ; d)$ и $\tilde{\tau}_{><}(\widetilde{E} ; d)$ во время пребывания мал. Как и ожидалось, эта зависимость аналогична представленной на рис. 3, она демонстрирует последовательность резонансов (некоторые различия можно объяснить тем фактом, что на рис. 3 представлены результаты, полученные из асимптотического выражения (36) для времени пребывания при $\left.\widetilde{E}_{i} \tilde{\sigma}^{2} \rightarrow \infty\right)$. При $\tilde{\sigma}=0.05$ вклады компонент $\widetilde{\tau}_{<}(\widetilde{E} ; d)$, движущихся в обратном направлении, и интерференционных членов $\tilde{\tau}_{><}(\widetilde{E} ; d)$ являются существенными, что можно видеть на рис. 5, значительно отличающемся от рис. 4. В этом случае зависимость от $\widetilde{U}$ времени пребывания характеризуется увеличением числа резонансов с бо́льшими амплитудами (однако значения амплитуд этих острых пиков не очень надежны вследствие ограниченной точности графика вблизи соответствующих значений $\widetilde{U})$. Такая картина может быть обусловлена увеличением области энергии (для рассматриваемого волнового пакета с широким распределением по энергии), которая вносит вклад в интеграл по $\widetilde{E}$ (случай $\tilde{\sigma}=1 / 3$ соответствует ситуации, когда энергия практически равна $\left.\widetilde{E}_{i}\right)$, а также влиянием компонент исходного волнового пакета, движущихся в обратном направлении. Такая картина соответствует представленной на рис. 2 зависимости, где показаны более сложная интерференционная картина в пространстве и более широкое распределение по времени в случае широкого (по распределению энергии) исходного волнового пакета в сравнении со случаем $\tilde{\sigma}=1 / 3$ (см. рис. 1$)$. 


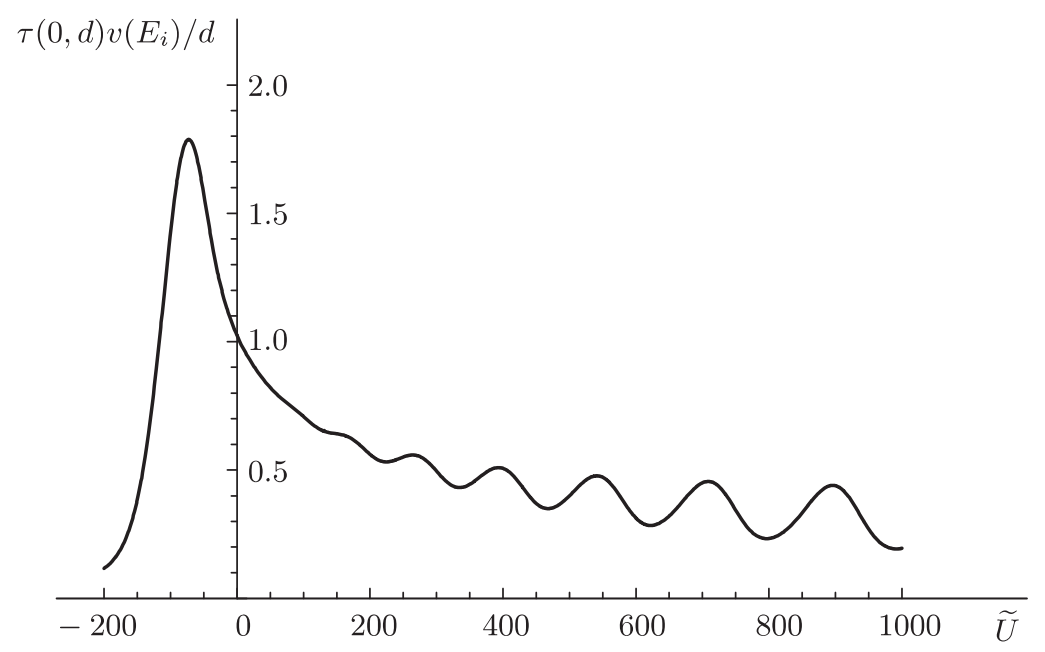

Рис. 4. Зависимость времени пребывания от амплитуды потенциальной ямы (барьера) для случая узкого распределения по энергии исходного волнового пакета $(\tilde{\sigma}=1 / 3)$.

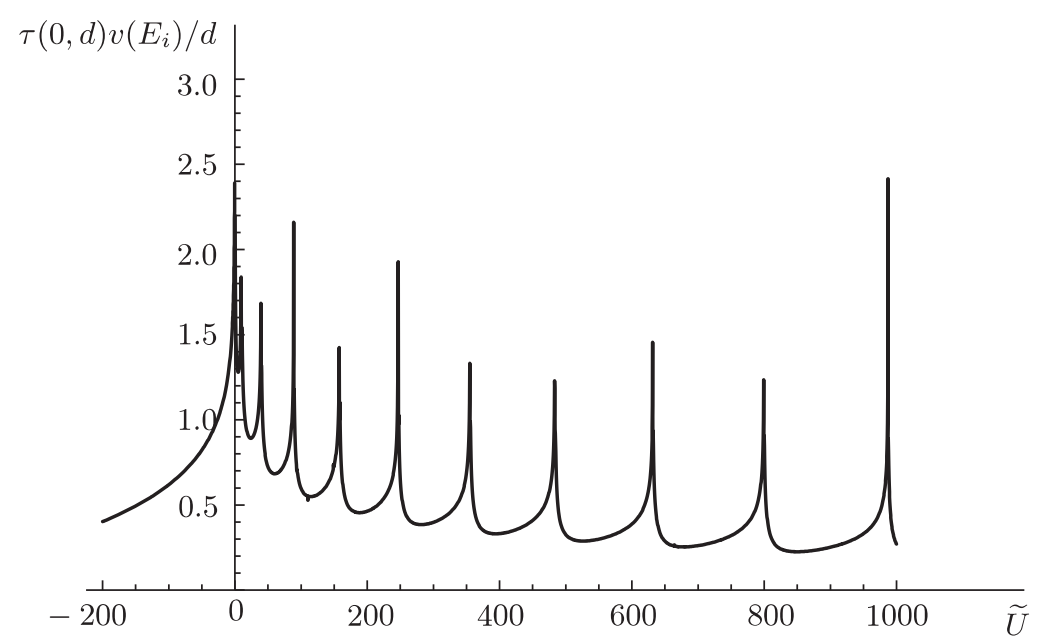

Рис. 5. Зависимость времени пребывания от амплитуды потенциальной ямы (барьера) для случая широкого распределения по энергии исходного волнового пакета $(\tilde{\sigma}=0.05)$. 


\section{5. ЗАКЛЮЧЕНИЕ}

Мы решили точно зависящее от времени уравнение Шредингера для частицы в присутствии одномерной прямоугольной потенциальной ямы (барьера). Решение (волновая функция частицы) представляет собой сумму слагаемых, соответствующих движению в прямом и обратном направлениях, при этом учитывается, что в общем случае исходный волновой пакет, заключенный в ограниченной области пространства и представляющий частицу, содержит как положительные, так и отрицательные компоненты импульса. Обе компоненты волновой функции выражаются в терминах интегралов от элементарных функций по полуограниченной области изменения энергии $E=0 \div \infty$ (в случае ямы связанные состояния не дают вклада в решение). Таким образом, плотность вероятности $|\psi(x, t)|^{2}$ нахождения частицы в точке $(x, t)$ пространства-времени, если исходно она была локализована в некоторой пространственной области и двигалась в некотором направлении, вообще говоря, определяется плотностью вероятности, соответствующей компоненте волнового пакета, движущейся в данном направлении $\left|\psi_{>}(x, t)\right|^{2}$, а также плотностями вероятности, связанными с компонентой, движущейся в противоположном направлении $\left|\psi_{>}(x, t)\right|^{2}$, и их интерференцией $2 \operatorname{Re}\left[\psi_{>}(x, t) \psi_{<}^{*}(x, t)\right]$. В случае, когда исходный волновой пакет является гауссовым, мы показали, что вклад компонент, движущихся в обратном направлении, в плотность вероятности $|\psi(x, t)|^{2}$ мал, если исходный волновой пакет имеет узкое распределение по энергии (импульсу), и получили асимптотические значения $\left|\psi_{>}(x, t)\right|^{2}$ в различных пространственных областях относительно области потенциальной ямы (барьера). Мы численно построили график точного результата для $|\psi(x, t)|^{2}$ в пространственной области ямы $(U>0)$ и показали, что компоненты волнового пакета, движущиеся в обратном направлении, существенно меняют плотность вероятности, если исходный волновой пакет является достаточно широким в энергетическом (импульсном) пространстве. Этот довольно парадоксальный квантово-механический результат проявляется в проблемах, связанных с измерением времени в квантово-механических эффектах.

Полученное решение применяется для вычисления времени прибытия и времени пребывания частицы в различных пространственных областях относительно области потенциальной ямы (барьера). Как и прежде, для узкого распределения по импульсам исходного волнового пакета аналитическое асимптотическое значение главного (в данном случае) члена, вносящего вклад во время пребывания в области потенциальной ямы (барьера), обусловлено компонентами плотности вероятности $\left|\psi_{>}(x, t)\right|^{2}$, соответствующими движению в прямом направлении. Оно было получено и представлено графически как функция амплитуды потенциала $U$, которая меняется от положительного значения (яма) до отрицательного (барьер). Чтобы показать, как компоненты, движущиеся в обратном направлении, и интерференционные компоненты плотности вероятности влияют на время пребывания, мы численно визуализировали точное выражение для этой величины как функцию амплитуды $U$ (значения которой меняются от положительных до отрицательных) как для узкого (когда вклад компонент, движущихся в обратном направлении, мал), так и для широкого (в энергетическом пространстве) исходного волнового пакета. В последнем случае наблюдается существенное изменение зависимости времени пребывания от амплитуды потенциала $U$. 
Кроме связи с фундаментальными проблемами квантовой механики, полученные результаты также могут быть важны для кинетической теории наноструктур, где рассмотренный нами прямоугольный потенциал (1) часто используется для моделирования потенциального профиля в многослойных структурах, например в приборах спинтроники. Полученные результаты для времени пребывания также могут представлять интерес в связи с проведенным недавно экспериментом, в котором исследуется время пересечения барьера, а также его интерпретацией, представленной в работе [16].

\section{Список литературы}

[1] A. D. Baute, I. L. Egusquiza, J. G. Muga, J. Phys. A, 34:20 (2001), 4289-4299.

[2] A. D. Baute, I. L. Egusquiza, J. G. Muga, Int. J. Theor. Phys. Group Theory Nonlinear Opt., 8:1 (2002), 1-17, arXiv: quant-ph/0007079.

[3] J. G. Muga, R. Sala Mayato, I. L. Egusquiza (eds.), Time in Quantum Mechanics, v.1, Lecture Notes in Physycs, 734, Springer, Berlin, 2008; J. G. Muga, A. Ruschhaupt, A. del Campo (eds.), Time in Quantum Mechanics, v.2, Lecture Notes in Physycs, 789, Springer, Berlin, 2009.

[4] M. N. Baibich, J. M. Broto, A. Fert, F. Nguyen Van Dau, F. Petroff, P. Etienne, G. Creuzet, A. Friederich, J. Chazelas, Phys. Rev. Lett., 61:21 (1988), 2472-2475.

[5] R. Julliere, Phys. Lett. A, 54:3 (1975), 225-226; P. LeClair, J. S. Moodera, R. Meservay, J. Appl. Phys., 76:10 (1994), 6546-6548.

[6] A. O. Barut, I. H. Duru, Phys. Rev. A, 38:11 (1988), 5906-5909.

[7] V.F. Los, A. V. Los, J. Phys. A, 43:5 (2010), 055304, 12 pp.

[8] V.F. Los, A. V. Los, J. Phys. A, 44:21 (2011), 215301, 16 pp.

[9] V.F. Los, M. V. Los, J. Phys. A, 45:9 (2012), 095302, 16 pp.

[10] Р. Фейнман, А. Хибс, Квантовая механика и интеграль по траекториям, Мир, М., 1968.

[11] J. G. Muga, S. Brouard, R. F. Snider, Phys. Rev. A, 46:9 (1992), 6075-6078.

[12] S. Cordero, G. Garcia-Calderón, J. Phys. A, 43:18 (2010), 185301, 18 pp.

[13] G. R. Allcock, Ann. Phys. (N. Y.), 53:2 (1969), 253-285; 286-310; 311-348.

[14] J. G. Muga, C. R. Leavens, Phys. Rep., 338:4 (2000), 353-438.

[15] M. Buttiker, Phys. Rev. B, 27:10 (1983), 6178-6188.

[16] E. A. Galapon, Phys. Rev. Lett., 108:17 (2012), 170402, 5 pp. 